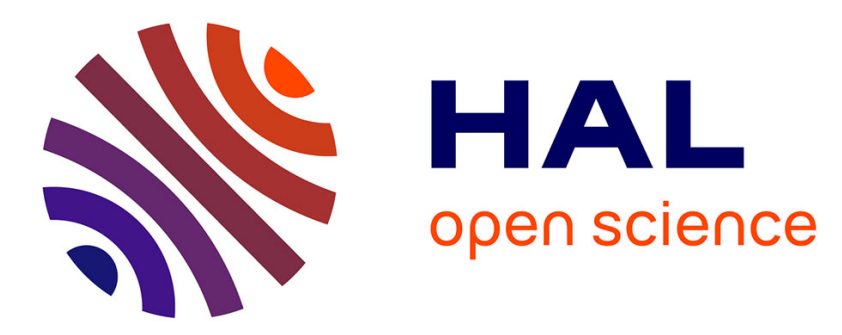

\title{
Mesoscopic magnetism and superconductivity
}

Ali C Basaran, Javier Villegas, J.S. Jiang, Axel Hoffmann, Ivan K Schuller

\section{To cite this version:}

Ali C Basaran, Javier Villegas, J.S. Jiang, Axel Hoffmann, Ivan K Schuller. Mesoscopic magnetism and superconductivity. MRS Bulletin, 2015, 40 (11), pp.925 - 932. 10.1557/mrs.2015.264 . hal-01841064

\section{HAL Id: hal-01841064 \\ https://hal.science/hal-01841064}

Submitted on 17 Jul 2018

HAL is a multi-disciplinary open access archive for the deposit and dissemination of scientific research documents, whether they are published or not. The documents may come from teaching and research institutions in France or abroad, or from public or private research centers.
L'archive ouverte pluridisciplinaire HAL, est destinée au dépôt et à la diffusion de documents scientifiques de niveau recherche, publiés ou non, émanant des établissements d'enseignement et de recherche français ou étrangers, des laboratoires publics ou privés. 


\title{
Mesoscopic magnetism and superconductivity
}

Ali C. Basaran ${ }^{1}$, Javier E. Villegas ${ }^{2}$, J.S. Jiang ${ }^{3}$, Axel Hoffmann ${ }^{4}$, and Ivan K. Schuller ${ }^{5}$

${ }^{1}$ Department of Physics and Center for Advanced Nanoscience, University of California-San Diego, USA; abasaran@physics.ucsd.edu

${ }^{2}$ Unité Mixte de Physique, CNRS, Thales, Univ. Paris-Sud, Université ParisSaclay, France; javier.villegas@cnrs-thales.fr

${ }^{3}$ Materials Science Division, Argonne National Laboratory, USA; jiang@anl.gov

${ }^{4}$ Materials Science Division, Argonne National Laboratory, USA;

hoffmann@anl.gov

${ }^{5}$ Department of Physics and Center for Advanced Nanoscience, University of California-San Diego, USA; ischuller@ucsd.edu

\begin{abstract}
Superconductivity and magnetism at intermediate ("mesoscopic") length scales between atomic and bulk, have a long history of interesting science. New science emerges due to the presence of multiple length scales, especially when these become comparable to relevant geometric sizes. New phenomena may appear due to topological interactions, geometric confinement, proximity between dissimilar materials, dimensional crossover, and collective effects induced by periodicity. In this review, we select a few, recent highlights that illustrate the type of novel science that can be accomplished in superconducting and magnetic structures. These materials can serve as model systems and provide new ideas, which can be extended to other systems such as ferroelectrics and multiferroics. We also highlight general open questions and new directions in which the field may move.
\end{abstract}

Work published in MRS Bulletin Volume 40, Issue 11 (Mesoscale Materials, Phenomena, and Functionality) November 2015 , pp. $925-932$

\section{https://doi.org/10.1557/mrs.2015.264}




\section{Introduction}

Magnetism and superconductivity provide excellent foundations for the development of novel ideas in materials physics and serve as examples for new mesoscopic science. Magnetic and superconducting (S) materials have a very interesting admixture of short and long length scales. The short, nanoscale (atomic, $<1 \mathrm{~nm}$ ) length scales, govern the ordering into specific crystalline structures and produce complex atomic-scale electronic interactions. It is probably safe to state that the appearance of superconductivity and magnetism in specific materials systems is often not well understood, and predicting specific, characteristic parameters such as the ordering temperatures, is even harder. Typical length scales that govern the appearance of superconductivity are given by the atomic arrangements of elements within a unit cell or the exchange length in a magnetic material. On the other hand, many superconducting and magnetic phenomena are governed by longer, mesoscopic (often 10 to 1000 times longer than interatomic distances) length scales. At these scales, there are well-defined predictions in agreement with experimental observations. Typical long length scales that appear in superconductivity are the coherence length and penetration depth and in magnetic materials are the dipolar and Ruderman-Kittel-KasuyaYosida length scales, all of which are longer than interatomic distances and independent of detailed atomic arrangements. ${ }^{\text {[ref] }}$

Many unique physical properties can be engineered into mesoscale artificial structures without the need for ultimate atomic perfection. In this article, we describe several general mesoscopic phenomena and illustrate them with an example from superconductivity or magnetism. Possible phenomena at mesoscopic length scales are categorized as topological, which depend on sample geometries; confinement, which are controlled by physical boundary conditions; proximity, in which close physical contact between dissimilar materials produces new effects; crossover, which is across different dimensions; and collective effects, which depend on some form of periodicity. Although we illustrate some of these phenomena in the fields of superconductivity and magnetism, the general concepts go beyond and can be extended to other physical phenomena such as 
ferroelectricity, elasticity, optics, and transport. Here, we specifically exclude phenomena that depend on detailed atomic arrangements, such as the appearance of superconductivity and magnetism at interfaces due to subtle atomic arrangements or bonding.

Figure 1 shows a comparison of length scales and structural characterization techniques. ${ }^{1}$ It is important to note that at the nanoscale $(<1 \mathrm{~nm})$, structural characterization techniques have difficulties in providing high-accuracy quantitative structural or chemical information. Whereas at longer, mesoscopic $(>1 \mathrm{~nm})$ length scales, there are many structural and chemical tools that provide quantitative measurements. Thus, at the mesoscale, structural and chemical issues are much less severe and hamper less pertinent to the understanding of the origin of unusual phenomena encountered.

Long magnetic and superconducting length scales have been known to produce mesoscopic phenomena as described in this article, see e.g., Table I. The full complexity and current research opportunities of mesoscale phenomena in magnetism ${ }^{2}$ can be illustrated by the behavior of spin waves ("magnons") in magnetic films. These fundamental excitations are due to the precession of the electron spin around an effective magnetic field, which may include internal fields, such as anisotropy fields, as well as externally applied fields. Besides the interaction with the effective magnetic field, the energy of the collective excitation is determined by the mutual interactions between spins, which includes short-scale exchange interactions, and mesoscopic-scale dipolar interactions. These dipolar interactions may stabilize inhomogeneous magnetic structures, since the energy gain from minimizing dipolar interactions may exceed the energy loss from exchange energies, which favor homogenous magnetic structures. Thus, by changing the magnon wavelength, it is possible to investigate the transition in the dynamics dominated by dipolar interactions (long wavelengths) to a regime dominated by exchange (short wavelengths).

Unique manifestations of mesoscopic physics can appear when long-range quantum coherent states, such as superconductivity, exist. The long superconducting coherence length produces unusual phenomena such as the well- 
known proximity effect in which a normal material may become superconducting by proximity to a superconductor. As a consequence, unique phenomena appear, such as the Josephson Effect, flow of electric current without any voltage applied in mesoscopic superconducting-normal-superconducting structures, and quantum coherent states in normal materials (Bohm-Aharonov effect) may be enhanced.

\section{Topology}

In many solid-state systems, interactions across different length scales are often responsible for developing or stabilizing states with distinct topologies. There are many examples of the formation of topological phases in superconductivity and magnetism, both artificially and naturally produced. These include systems such as magnetic and superconducting vortices, skyrmions, vortex arrays, Majorana fermions, and topological superconductors. The interaction with artificially structured materials in unusual topologies produce unique physics beyond what is found in naturally occurring systems.

\section{Magnetic skyrmions}

In magnetic systems, a wide variety of magnetic structures with distinct topology exist, which can exhibit quasiparticle-like behavior. ${ }^{18}$ Well-studied examples are magnetic vortices, ${ }^{13,14}$ stabilized by long-range dipolar interactions that exhibit collective motion governed by the dynamics of the individual spins forming the vortex. ${ }^{19,20}$ Recently, magnetic skyrmions ${ }^{21}$ have garnered increasing interest. These are topologically distinct spin structures, in which the spins point in all three-dimensional directions, as is schematically shown in Figure 2a. Magnetic skyrmions were discovered ${ }^{21}$ in bulk materials with chiral Dzyaloshinskii-Moriya interactions (DMI), which is an antisymmetric exchange interaction given by the vector cross product of two neighboring spins. At temperatures close to the magnetic ordering temperature and moderately high magnetic fields, the competition between the DMI (favoring a helical magnetic structure) and direct interactions with externally applied magnetic fields (favoring a preferred magnetization direction) results in a compromise, where tubes of magnetization form that are aligned antiparallel to that of the surrounding matrix. Unlike 
magnetic vortices, which occur in geometrically confined structures, magnetic skyrmions can form in extended bulk materials. Often, they form a well-defined hexagonal lattice similar to vortex-lattices in superconductors. Just like superconducting vortices, skyrmions in magnetic systems are mobile and can be moved with very small electric currents. ${ }^{22,23}$

The efficient manipulation of skyrmions with small electric currents has two origins. First, electrons that move in a skyrmion spin texture experience a virtual magnetic field (exceeding $100 \mathrm{~T}$ for skyrmions with diameters in the 10 $\mathrm{nm}$ range and below) that results in their transverse deflection and gives rise to the topological Hall effect. ${ }^{23,24}$ At the same time, a back-action results in motion of the skyrmions. The second aspect that facilitates skyrmion motion is decreased pinning due to their compact structure, which allows them to move around defects. Since skyrmions can be small (few $\mathrm{nm}$ ) and easily manipulated electrically, they are potentially useful for information technologies. ${ }^{25}$

To realize the ambitious goal of utilizing magnetic skyrmions for information technologies, efficient generation and annihilation of individual skyrmions on demand is needed. For instance, in thin FePd on $\operatorname{Ir}(111)$, skyrmions can be generated and annihilated via spin-polarized currents at cryogenic temperatures $(8 \mathrm{~K}) .{ }^{16}$ For practical applications, however, generation of skyrmions at room temperature and demonstration of high mobility is needed. This has recently been demonstrated in $\mathrm{Ta} / \mathrm{CoFeB} / \mathrm{TaO}_{x}$ multilayers, as shown in Figure $2 \mathrm{~b} .{ }^{15}$ The perpendicular anisotropy in this material results in extended magnetic band domains, as seen on the left-hand side of Figure 2b. At the same time, a heavy element (e.g., Ta) layer in combination with a thin ferromagnetic (FM) layer with perpendicular anisotropy may stabilize domain walls with welldefined chirality due to interfacial DMI. ${ }^{26,27}$ These can be very efficiently moved due to the spin Hall effect. ${ }^{28}$ In the device shown in Figure $2 b$, this electric current-driven domain-wall motion is used to push the band domain through the constriction. Upon exiting from the constriction, the band domain expands laterally due to laterally inhomogeneous spin transfer torque giving rise to an instability, generating individual skyrmion bubbles [see Figure 2c]. 
This mechanism is similar to Rayleigh-Plateau instabilities in surfacetension-dominated fluid flows, which result in droplet formation, i.e., for a dripping water faucet. ${ }^{29}$ Interestingly, the critical electrical current for the motion of these synthetically produced skyrmions is two orders of magnitude smaller than for regular domain wall motion, indicating that indeed the peculiar structure of skyrmions results in significantly decreased pinning. ${ }^{15}$

\section{Superconducting ratchets}

Superconducting vortices interacting with artificial pinning arrays have attracted attention for over two decades ${ }^{30,31}$ and yielded unique phenomena such as vortex ratchets. In a superconducting ratchet, a periodic alternating current is rectified giving rise to a DC voltage. The topology that produces this effect consists of a superconducting $(\mathrm{Nb}$, for instance) film on top of a rectangular array of triangular pinning sites, as shown in Figure 3. ${ }^{32}$ Upon depinning under a symmetric AC driving current, vortices acquire a net velocity that depends on their number (i.e., on the perpendicular applied magnetic field). Up to three vortices per unit cell of the array are trapped in the triangles and acquire a net velocity in the same direction (red arrows) when depinned [see Figs. 3(a) and (b)]. For higher-vortex densities, the excess vortices become interstitial (blue), and "feel" the opposite asymmetry via their interaction with the (red) pinned vortices [see Figs. 3(c) and (d)]. Under an AC driving force, interstitials depin first and acquire a net velocity opposite (blue arrow) to the pinned ones (red). The reversed rectification holds until a sufficiently intense drive depins vortices trapped by triangles, producing positive rectification. Thus, the system behaves as a very unique electrical rectifier, in which an injected AC current leads to a DC voltage whose sign depends on the applied magnetic field and the magnitude of current.

Over the past decade, vortex ratchets have been profusely studied, (e.g., seeking new types of asymmetric pinning [nonuniform arrays, constrictions, surface barriers], high-frequency effects). ${ }^{33,34}$ Recent developments in the area, including reconfigurable energy landscapes, ${ }^{35,36}$ vortex manipulation through magnetic force microscopy, ${ }^{37}$ and single-vortex imaging, ${ }^{37,38}$ have provided key ingredients and paved the way toward novel vortex ratchet applications. At the 
same time, vortices on artificial pinning arrays constitute a much broader research area whose recent developments and exciting prospects cannot be detailed here. Theory ${ }^{39}$ and experiments ${ }^{40}$ have shown that nonperiodic arrays provide sizable superconducting critical current enhancements over a larger field range than those obtained with periodic arrays. Thus, ordered, nonperiodic arrays are probably the route toward new developments in the field.

\section{Proximity}

Mesoscopic length scales clearly manifest themselves in situations when two dissimilar materials are in close physical proximity. In many cases, there are general so-called proximity effects in which the interactions between competing

phenomena produce new physics. Examples of such long-range effects, which are independent of detailed atomic arrangements, include the classical superconducting or magnetic proximity effect in which a normal material may become superconducting or magnetic, and asymmetric magnetic reversal in FM/antiferromagnetic (AFM) bilayers.

\section{FM heterostructures}

FM heterostructures are classic examples in which mesoscopic phenomena appear due to competition between various magnetic length scales. The typical magnetic domain wall width is larger than several tens of $\mathrm{nm}$ in many FM materials. It is very unusual and unexpected to find a small $(<10 \mathrm{~nm})$ domain wall, especially in a typical soft ferromagnet, such as Permalloy or Yttrium Iron Garnet (YIG). A classic interesting example from the fundamental and applied points of view, where very short domain walls can be engineered, is the one formed by combinations of magnetically soft/hard bilayers. ${ }^{41-44}$ In particular, an FM in intimate contact with an $\mathrm{AFM}^{45}$ or a soft magnetic material in contact with a hard one exhibits these interesting effects. Due to the interfacial interactions in FM/AFM ("exchange biased") or soft/hard ("FM spring") heterostructures, a short mesoscopic domain wall $(<10 \mathrm{~nm})$ can form, as shown in Figure 4a. As a consequence, the FM reversal becomes asymmetric and reversible as shown in Figure $4 \mathrm{~b} .{ }^{46}$ In these systems, the magnetization reversal initiates with the 
nucleation of a quasi-Bloch wall, where the magnetization rotates in the soft FM in a plane parallel to the interface. This results in a spiral spin configuration that motivates the expression 'exchange spring'. As the reversed field increases, the quasi-Bloch wall in the soft FM is compressed against the hard one, and the interfacial hard spins also increasingly rotate. The soft spins rotate back into alignment with the hard phase if the reverse field is removed.

Nucleation and evolution of the quasi-Bloch walls during exchange-spring reversal have been characterized using a magneto-optical indicator, ${ }^{47}$ polarized neutron reflectivity, ${ }^{48}$ coherent nuclear resonant scattering, ${ }^{49} \mathrm{x}$-ray resonant magnetic scattering, ${ }^{50}$ and layer-resolved conversion-electron Mössbauer spectroscopy, ${ }^{51}$ and magnetotransport. ${ }^{52}$

It is important to note that formation of mesoscopic quasi-Bloch walls are mainly governed by the interfacial coupling between soft/ hard or FM/AFM, which depends on the microstructural details of the interface and sample morphology. This can produce many rich phenomena ${ }^{53}$ related to roughness, ${ }^{54,55}$ grain size,${ }^{56-58}$ crystallinity, ${ }^{59,60}$ interlayer diffusion, ${ }^{61}$ and defects. ${ }^{62-64}$ Furthermore, these properties depend on temperature and magnetic field history of the sample. ${ }^{65,66}$ Therefore, general magnetic properties on these heterostructures are attributable to several extrinsic and intrinsic parameters, which become very important at the mesoscale. ${ }^{67-69}$

\section{Triplet superconductivity}

The superconducting $(\mathrm{S})$ proximity effect — penetration of the superconducting condensate into a nonsuperconducting material in contact with a superconductor - is long-ranged (up to microns) for clean metals. However, it is usually short-ranged for FMs, because the exchange field tends to align the electron spins breaking apart conventional opposite-spin singlet Cooper pairs. ${ }^{70}$ This occurs within a length scale $\xi_{\mathrm{F}} \sim \mathrm{nm}$, with $\xi_{\mathrm{F}}=\sqrt{\hbar D / 2 E_{\mathrm{ex}}}$ for diffusive systems ( $D$ is the electronic diffusion constant, $E_{\mathrm{ex}}$ is the exchange splitting, and $\hbar$ is the reduced Planck constant) and $\xi_{\mathrm{F}}=\nu_{\mathrm{F}} / 2 E_{\mathrm{ex}}$ for ballistic systems ( $\nu_{\mathrm{F}}$ is the Fermi velocity). 
Long-range proximity effects have been experimentally observed at specific S/FM interfaces ${ }^{71-76}$ and explained by the generation of unconventional equal-spin triplet pairs. These are immune to the exchange field and can propagate over long distances into the FM. ${ }^{77-79}$ While the salient features of conventional S/FM proximity effects have received attention for two decades, ${ }^{70}$ equal-spin triplets currently attract the spotlight. Although triplets were earlier evoked to explain the coexistence of ferromagnetism and superconductivity in certain bulk materials, ${ }^{80}$ artificial S/FM hybrids have only recently opened the door to deeper understanding and manipulation of this unconventional superconducting state.

Open research topics in this area include an understanding of specific systems (e.g., cuprate/manganite interfaces ${ }^{75,76,81}$ ) and the search for new S/FM combinations and novel mechanisms. Theory has recently shown that spin-orbit coupling ${ }^{82}$ can promote equal-spin triplet pairing, a possibility that opens an approach to manipulate the triplet condensate. The dynamic coupling between the equal-spin triplet condensate and the FM magnetization is another appealing perspective. Theory shows that a time-varying magnetization influences the triplet condensate generation due to the non-equilibrium spin-accumulation induced at the interface ${ }^{83,84}$ Conversely, an equal-spin triplet supercurrent is predicted to affect the magnetization via spin-torque ${ }^{85,86}$ However, no experimental studies exist in which the triplet condensate is manipulated with stimuli such as microwaves. These fundamental prospects, in addition to the potential of triplet superconductivity for "superconducting spintronics," make this emergent topic an exciting area of research.

\section{Outlook and future}

The outlook for future research on mesoscopic behavior in magnets and superconductors is very bright. The synthesis and control of artificial structures in different, complex materials allows control across length scales, which are relevant for the development of novel physics. It is especially interesting that long-range coherent behavior allows for the study of quantum phenomena at room temperature, such as Bose-Einstein condensation of magnons. At the same time, it 
opens up a series of important issues, questions, and potential capabilities for new physics.

\section{Magnon Bose-Einstein condensation}

Interestingly, in thin films, the competition between short-range exchange and long-range dipolar interactions can give rise to nonmonotonic magnon dispersion with minima at finite wave vectors, schematically shown in Figure 5a. ${ }^{87}$ These minima can be utilized to explore collective magnon states, which can form a Bose-Einstein condensate (BEC) even at room temperature, as initially demonstrated a decade ago ${ }^{88}$ based on earlier theoretical predictions. ${ }^{89}$ In this seminal experiment, a very dense gas of magnons was excited via parametric pumping to an energy well above the magnon dispersion minimum. During parametric pumping, a microwave photon generates two magnons with opposite wave vectors, as shown schematically in Figure 5a. Subsequently, four magnonscattering processes redistribute the energy, while maintaining the magnon density. Once the magnon density exceeds a critical value, the BEC forms at the dispersion curve minimum, as experimentally measured with spatially resolved Brillouin light-scattering. ${ }^{87,88,90,91}$ The coherence between the two components of the BEC with opposite wave vectors produces a standing wave pattern directly revealing the magnon wavelength at the dispersion minima.

The generation of room-temperature magnon BECs opens up exciting opportunities for the exploration of macroscopic quantum coherent phenomena at temperatures far above those studied in any superconductors or liquid He. For example, Josephson effects, between two superconducting condensates coupled via a weak link, have been key to high-precision measurements, as well as the practical implementations of quantum computation. Interestingly, although Josephson effects have been studied in superconducting systems for about half a century, only recently has this concept been extended to other BEC systems such as atomic gases. ${ }^{92}$ A recent theoretical prediction claims that similar Josephson effects should exist for magnon BECs. ${ }^{93}$ One main challenge is to generate magnon BECs with spatially modulated phase relationships. Toward this end, establishing magnon BEC via local charge ${ }^{94}$ or heat currents ${ }^{95}$ may offer a 
possible solution. In fact, it was suggested very recently that temperature gradients within magnon BECs may be accompanied by magnon supercurrents. ${ }^{96}$ Other approaches of spatially tailoring magnon properties include local field modulations. ${ }^{97,98}$

\section{Superconducting spintronics}

Conventional spintronics combines information storage in FMs (memory) and information transport by spin-polarized electrons. The archetypal device (spinvalve) consists of two FM electrodes connected by a nonmagnetic channel. The relative orientation of the electrodes' magnetization (parallel/antiparallel) yields electrical-resistance states (low/high, respectively), due to spin-dependent electron scattering. The superconducting analogues of the spin-valve generally consist of S/FM hybrids that show resistive switching driven by the magnetic history, ${ }^{99-107}$ using the dependence of the critical temperature $\left(T_{\mathrm{C}}\right)$ on the FM magnetization. However, many superconducting "spin-valves" do not truly exploit spin-dependent transport, rather they use a variety of different mechanisms, from FM stray magnetic field ${ }^{102,104,108,109}$ or exchange field ${ }^{99,105}$ effects on the superconductor, to the proximity effect-either the conventional (short-range singlet) ${ }^{101}$ or triplet ${ }^{107,110,111}$ (see example in Figure 6). Only in some cases $^{100,103}$ does resistive switching arise from spin accumulation in the superconductor due to quasiparticle injection from the ferromagnet. Regardless of the governing mechanism, resistance switching is usually much larger than in conventional spin-valves. However, as $T_{\mathrm{C}}$ variations are minute, the "spin-valve" effect is observed only in a very narrow temperature range near the intrinsic $T_{\mathrm{C}}$ [see Figure 6c].

A conceptual leap uses superconductivity to improve "information transport" above the conventional "spin-valves" (which work as a memory). For this, superconductors (instead of metals or semiconductors) are used as the spintransport channel. Interestingly, experiments have repeatedly shown that the spindiffusion lengths in superconducting metals are significantly longer below $T_{\mathrm{C}}$ than above $T_{\mathrm{C}},{ }^{12-114}$ due to different spin-relaxation mechanisms. Equal-spin triplet superconductivity can be used to induce "spin-polarized superconductivity" in the 
FM, as opposed to creating a spin accumulation in the superconductor. Therefore, information would be carried by spin-polarized superconducting pairs in the FM, which leads to important implications.

In contrast to the nonequilibrium spin accumulation produced by spin injection, equal-spin triplet superconductivity is an equilibrium state induced by proximity in the FM without any external stimuli (such as electrical current, microwaves, or light). This should allow for novel, simpler device architectures. Also, certain quantum coherence effects (e.g., nonlocality and Josephson effects) provide possibilities ${ }^{115,116}$ that are absent in conventional spintronics. Recent realizations seeking that potential include magnetic-history switchable Josephson junctions. ${ }^{117}$ Of course, there are quite a few challenges ahead. For instance, devising methods that efficiently discriminate and "read" the polarization of equal-spin triplet states in FMs, when both spin-up and spin-down triplet pairs coexist. A possible way is the use of half-FM metals, in which only one equalspin polarization is possible. Another standing challenge that could attract future attention is the transfer of the equal-spin triplets from the FM into another material, (e.g., a nonmagnetic metal) where equal-spin triplets could stay phase coherent over long distances.

\section{Conclusions}

The properties of materials at mesoscopic length scales provide a unique opportunity for interesting basic research studies and for the development of unique, useful devices. The long length scales (larger than interatomic spaces) of mesoscopic structures allow the pursuit of many studies without additional complications related to synthesis and characterization of structurally perfect materials. Nevertheless, it is important to be vigilant to avoid complications due to extrinsic effects. In many mesoscopic systems, it is precisely the imperfections that make the science interesting. In particular, in the field of magnetism and superconductivity, there are many phenomena that depend on a complicated interplay between long mesoscopic and atomic length scales. Because of the vast number of length scales existing in these materials and the broad area impacted, it 
is impossible to have a comprehensive review in which all different aspects of these are covered.

\section{Acknowledgements}

This is a highly collaborative review. The outline of this article was conceived jointly, extensively debated, and the article was written by multiple iterations between all the coauthors. The research at UCSD was supported by the Office of Basic Energy Science, US Department of Energy, BES-DMS funded by the Department of Energy's Office of Basic Energy Science, DMR under grant DE FG02 87ER-45332 and the US AFOSR Grant No. FA9550-12-1-0381. The work at Argonne was supported by the US Department of Energy, Office of Science, Basic Energy Sciences, Materials Sciences and Engineering Division. Work at CNRS/Thales supported by ERC grant No. 647100 "SUSPINTRONICS". I.K.S. thanks the US Department of Defense for a National Security Science and Engineering Faculty Fellowship (NSSEFF). We thank Y. Bruynseraede for work on the initial stages of this manuscript.

\section{References}

1. I.K. Schuller, S. Kim, C. Leighton, J. Magn. Magn. Mater. 200 (1-3), 571 (1999).

2. A. Hoffmann, H. Schultheiß, Curr. Opin. Solid State Mater. Sci. 19 (4), 253 (2015).

3. Y. Aharonov, D. Bohm, Phys. Rev. 115 (3), 485 (1959).

4. V.T. Petrashov, V.N. Antonov, P. Delsing, R. Claeson, Phys. Rev. Lett. 70 (3), 347 (1993).

5. E.N. Bogachek, I.A. Romanovsky, U. Landman, Phys. Rev. B Condens. Matter 78 (17), 174515 (2008).

6. S.T. Ruggiero, T.W. Barbee, M.R. Beasley, Phys. Rev. Lett. 45 (15), 1299 (1980).

7. C.S.L. Chun, G.-G. Zheng, J.L. Vincent, I.K. Schuller, Phys. Rev. B Condens. Matter 29 (9), 4915 (1984). 
8. S.M. Mohseni, S.R. Sani, J. Persson, T.N.A. Nguyen, S. Chung, Y. Pogoryelov, P.K. Muduli, E. Iacocca, A. Eklund, R.K. Dumas, S. Bonetti, A. Deac, M.A. Hoefer, J. Åkerman, Science 339 (6125), 1295 (2013).

9. A.M. Kosevich, B.A. Ivanov, A.S. Kovalev, Phys. Rep. 194 (3-4), 117 (1990).

10. J.I. Martín, M. Vélez, J. Nogués, I.K. Schuller, Phys. Rev. Lett. 79 (10), 1929 (1997).

11. B.D. Josephson, Phys. Lett. 1 (7), 251 (1962).

12. C. Visani, Z. Sefrioui, J. Tornos, C. Leon, J. Briatico, M. Bibes, A. Barthelemy, J. Santamaria, J.E. Villegas, Nat. Phys. 8 (7), 539 (2012).

13. R.P. Cowburn, D.K. Koltsov, A.O. Adeyeye, M.E. Welland, D.M. Tricker, Phys. Rev. Lett. 83 (5), 1042 (1999).

14. T. Shinjo, T. Okuno, R. Hassdorf, K. Shigeto, T. Ono, Science 289 (5481), 930 (2000).

15. W. Jiang, P. Upadhyaya, W. Zhang, G. Yu, M.B. Jungfleisch, F.Y. Fradin, J.E. Pearson, Y. Tserkovnyak, K.L. Wang, O. Heinonen, S.G.E. te Velthuis, A. Hoffmann, Science 349 (6245), 283 (2015).

16. N. Romming, C. Hanneken, M. Menzel, J.E. Bickel, B. Wolter, K. von Bergmann, A. Kubetzka, R. Wiesendanger, Science 341 (6146), 636 (2013).

17. J.O. Vasseur, L. Dobrzynski, B. Djafari-Rouhani, H. Puszkarski, Phys. Rev. B Condens. Matter 54 (2), 1043 (1996).

18. H.B. Braun, Adv. Phys. 61 (1), 1 (2012).

19. S.B. Choe, Y. Acremann, A. Scholl, A. Bauer, A. Doran, J. Stohr, H.A. Padmore, Science 304 (5669), 420 (2004).

20. V. Novosad, F.Y. Fradin, P.E. Roy, K.S. Buchanan, K.Y. Guslienko, S.D. Bader, Phys. Rev. B Condens. Matter 72 (2), 024455 (2005).

21. S. Mühlbauer, B. Binz, F. Jonietz, C. Pfleiderer, A. Rosch, A. Neubauer, R. Georgii, P. Böni, Science 323 (5916), 915 (2009).

22. T. Schulz, R. Ritz, A. Bauer, M. Halder, M. Wagner, C. Franz, C. Pfleiderer, K. Everschor, M. Garst, A. Rosch, Nat. Phys. 8 (4), 301 (2012).

23. K. Everschor-Sitte, M. Sitte, J. Appl. Phys. 115 (17), 172602 (2014).

24. A. Neubauer, C. Pfleiderer, B. Binz, A. Rosch, R. Ritz, P.G. Niklowitz, P. Boni, Phys. Rev. Lett. 102 (18), 186602 (2009). 
25. A. Fert, V. Cros, J. Sampaio, Nat. Nanotechnol. 8 (3), 152 (2013).

26. S. Emori, U. Bauer, S.-M. Ahn, E. Martinez, G.S.D. Beach, Nat. Mater. 12 (7), 611 (2013).

27. K.S. Ryu, L. Thomas, S.H. Yang, S. Parkin, Nat. Nanotechnol. 8 (7), 527 (2013).

28. A. Hoffmann, IEEE Trans. Magn. 49 (10), 5172 (2013).

29. J. Eggers, Rev. Mod. Phys. 69 (3), 865 (1997).

30. M. Baert, V.V. Metlushko, R. Jonckheere, V.V. Moshchalkov, Y. Bruynseraede, Phys. Rev. Lett. 74 (16), 3269 (1995).

31. J.I. Martin, M. Velez, J. Nogues, I.K. Schuller, Phys. Rev. Lett. 79 (10), 1929 (1997).

32. J.E. Villegas, S. Savel'ev, F. Nori, E.M. Gonzalez, J.V. Anguita, R. García, J.L. Vicent, Science 302 (5648), 1188 (2003).

33. J. Van de Vondel, V.N. Gladilin, A.V. Silhanek, W. Gillijns, J. Tempere, J.T. Devreese, V.V. Moshchalkov, Phys. Rev. Lett. 106 (13), 137003 (2011).

34. R. Wordenweber, E. Hollmann, J. Schubert, R. Kutzner, G. Panaitov, Phys. Rev. B Condens. Matter 85 (6), 064503 (2012).

35. A. Crassous, R. Bernard, S. Fusil, K. Bouzehouane, D. Le Bourdais, S. Enouz-Vedrenne, J. Briatico, M. Bibes, A. Barthelemy, J.E. Villegas, Phys. Rev. Lett. 107 (24), 247002 (2011).

36. J. Trastoy, M. Malnou, C. Ulysse, R. Bernard, N. Bergeal, G. Faini, J. Lesueur, J. Briatico, J.E. Villegas, Nat. Nanotechnol. 9 (9), 710 (2014).

37. O.M. Auslaender, L. Luan, E.W.J. Straver, J.E. Hoffman, N.C. Koshnick, E. Zeldov, D.A. Bonn, R. Liang, W.N. Hardy, K.A. Moler, Nat. Phys. 5 (1), 35 (2009).

38. L. Embon, Y. Anahory, A. Suhov, D. Halbertal, J. Cuppens, A. Yakovenko, A. Uri, Y. Myasoedov, M.L. Rappaport, M.E. Huber, A. Gurevich, E. Zeldov, Sci. Rep.-UK 5, 7598 (2015).

39. D. Ray, C.J.O. Reichhardt, B. Jankó, C. Reichhardt, Phys. Rev. Lett. 110 (26), 267001 (2013).

40. S. Guénon, Y.J. Rosen, A.C. Basaran, I.K. Schuller, Appl. Phys. Lett. 102 (25), 252602 (2013). 
41. E.E. Fullerton, J.S. Jiang, M. Grimsditch, C.H. Sowers, S.D. Bader, Phys. Rev. B Condens. Matter 58 (18), 12193 (1998).

42. P. Steadman, M. Ali, A.T. Hindmarch, C.H. Marrows, B.J. Hickey, S. Langridge, R.M. Dalgliesh, S. Foster, Phys. Rev. Lett. 89 (7), 077201 (2002).

43. Y. Henry, S. Mangin, T. Hauet, F. Montaigne, Phys. Rev. B Condens. Matter 73 (13), 134420 (2006).

44. G.H. Guo, G.F. Zhang, X.G. Wang, J. Appl. Phys. 108 (4), 043919 (2010).

45. R. Morales, Z.-P. Li, O. Petracic, X. Batlle, I.K. Schuller, J. Olamit, K. Liu, Appl. Phys. Lett. 89 (7), 072504 (2006).

46. R. Morales, A.C. Basaran, J.E. Villegas, D. Navas, N. Soriano, B. Mora, C. Redondo, X. Batlle, I.K. Schuller, Phys. Rev. Lett. 114 (9), 097202 (2015).

47. V.S. Gornakov, V.I. Nikitenko, A.J. Shapiro, R.D. Shull, J.S. Jiang, S.D. Bader, J. Magn. Magn. Mater. 246 (1-2), 80 (2002).

48. K.V. O’Donovan, J.A. Borchers, C.F. Majkrzak, O. Hellwig, E.E. Fullerton, Phys. Rev. Lett. 88 (6), 067201 (2002).

49. R. Rohlsberger, H. Thomas, K. Schlage, E. Burkel, O. Leupold, R. Ruffer, Phys. Rev. Lett. 89 (23), 237201 (2002).

50. Y. Choi, J.S. Jiang, J.E. Pearson, S.D. Bader, J.P. Liu, Appl. Phys. Lett. 91 (2), 022502 (2007).

51. V.M. Uzdin, A. Vega, A. Khrenov, W. Keune, V.E. Kuncser, J.S. Jiang, S.D. Bader, Phys. Rev. B Condens. Matter 85 (2), 024409 (2012).

52. A.C. Basaran, R. Morales, S. Guénon, I.K. Schuller, Appl. Phys. Lett. 106 (25), 252404 (2015).

53. J. Nogués, I.K. Schuller, J. Magn. Magn. Mater. 192 (2), 203 (1999).

54. W. Kuch, L.I. Chelaru, F. Offi, J. Wang, M. Kotsugi, J. Kirschner, Nat. Mater. 5 (2), 128 (2006).

55. J. Nogués, D. Lederman, T.J. Moran, I.K. Schuller, K.V. Rao, Appl. Phys. Lett. 68 (22), 3186 (1996).

56. T. Saerbeck, H. Zhu, D. Lott, H. Lee, P.R. LeClair, G.J. Mankey, A.P.J. Stampfl, F. Klose, J. Appl. Phys. 114 (1), 013901 (2013).

57. K. Nishioka, C. Hou, H. Fujiwara, R.D. Metzger, J. Appl. Phys. 80 (8), 4528 (1996). 
58. M. Pakala, Y. Huai, G. Anderson, L. Miloslavsky, J. Appl. Phys. 87 (9), 6653 (2000).

59. J. Nogués, T.J. Moran, D. Lederman, I.K. Schuller, K.V. Rao, Phys. Rev. B Condens. Matter 59 (10), 6984 (1999).

60. R. Jungblut, R. Coehoorn, M.T. Johnson, J. aan de Stegge, A. Reinders, J. Appl. Phys. 75 (10), 6659 (1994).

61. Y.K. Kim, S.-R. Lee, S.A. Song, G.-S. Park, H.S. Yang, K.-I. Min, J. Appl. Phys. 89 (11), 6907 (2001).

62. A. Mougin, T. Mewes, M. Jung, D. Engel, A. Ehresmann, H. Schmoranzer, J. Fassbender, B. Hillebrands, Phys. Rev. B Condens. Matter 63 (6), 060409 (2001).

63. J.-V. Kim, R.L. Stamps, Appl. Phys. Lett. 79 (17), 2785 (2001).

64. A.C. Basaran, T. Saerbeck, J. de la Venta, H. Huckfeldt, A. Ehresmann, I.K. Schuller, Appl. Phys. Lett. 105 (7), 072403 (2014).

65. J. Nogués, D. Lederman, T.J. Moran, I.K. Schuller, Phys. Rev. Lett. 76 (24), 4624 (1996).

66. Z.-P. Li, J. Eisenmenger, C.W. Miller, I.K. Schuller, Phys. Rev. Lett. 96 (13), 137201 (2006).

67. C.L. Dennis, R.P. Borges, L.D. Buda, U. Ebels, J.F. Gregg, M. Hehn, E. Jouguelet, K. Ounadjela, I. Petej, I.L. Prejbeanu, M.J. Thornton, J. Phys. Condens. Matter 14 (49), R1175 (2002).

68. J.I. Martín, J. Nogués, K. Liu, J.L. Vicent, I.K. Schuller, J. Magn. Magn. Mater. 256 (1-3), 449 (2003).

69. J. Nogués, J. Sort, V. Langlais, V. Skumryev, S. Suriñach, J.S. Muñoz, M.D. Baró, Phys. Rep. 422 (3), 65 (2005).

70. A.I. Buzdin, Rev. Mod. Phys. 77 (3), 935 (2005).

71. R.S. Keizer, S.T.B. Goennenwein, T.M. Klapwijk, G.X. Miao, G. Xiao, A. Gupta, Nature 439 (7078), 825 (2006).

72. T.S. Khaire, M.A. Khasawneh, W.P. Pratt Jr., N.O. Birge, Phys. Rev. Lett. 104 (13), 137002 (2010).

73. D. Sprungmann, K. Westerholt, H. Zabel, M. Weides, H. Kohlstedt, Phys. Rev. B Condens. Matter 82 (6), 060505 (2010).

74. J.W.A. Robinson, J.D.S. Witt, M.G. Blamire, Science 329 (5987), 59 (2010). 
75. Y. Kalcheim, T. Kirzhner, G. Koren, O. Millo, Phys. Rev. B Condens. Matter 83 (6), 064510 (2011).

76. C. Visani, Z. Sefrioui, J. Tornos, C. Leon, J. Briatico, M. Bibes, A. Barthelemy, J. Santamaria, J.E. Villegas, Nat. Phys. 8 (7), 539 (2012).

77. F.S. Bergeret, A.F. Volkov, K.B. Efetov, Phys. Rev. Lett. 86 (18), 4096 (2001).

78. A. Kadigrobov, R.I. Shekhter, M. Jonson, Europhys. Lett. 54 (3), 394 (2001).

79. M. Eschrig, Nat. Phys. 5 (6), 384 (2009).

80. S.S. Saxena, P. Agarwal, K. Ahilan, F.M. Grosche, R.K.W. Haselwimmer, M.J. Steiner, E. Pugh, I.R. Walker, S.R. Julian, P. Monthoux, G.G. Lonzarich, A. Huxley, I. Sheikin, D. Braithwaite, J. Flouquet, Nature 406 (6796), 587 (2000).

81. M. Egilmez, J.W.A. Robinson, J.L. MacManus-Driscoll, L. Chen, H. Wang, M.G. Blamire, Europhys. Lett. 106 (3), 37003 (2014).

82. F.S. Bergeret, I.V. Tokatly, Phys. Rev. Lett. 110 (11), 117003 (2013).

83. M. Houzet, Phys. Rev. Lett. 101 (5), 057009 (2008).

84. T. Yokoyama, Y. Tserkovnyak, Phys. Rev. B Condens. Matter 80 (10), 104416 (2009).

85. J. Linder, A. Brataas, Z. Shomali, M. Zareyan, Phys. Rev. Lett. 109 (23), 237206 (2012).

86. N.G. Pugach, A.I. Buzdin, Appl. Phys. Lett. 101 (24), 242602 (2012).

87. P. Nowik-Boltyk, O. Dzyapko, V.E. Demidov, N.G. Berloff, S.O.

Demokritov, Sci. Rep. 2, 482 (2012).

88. S.O. Demokritov, V.E. Demidov, O. Dzyapko, G.A. Melkov, A.A. Serga, B. Hillebrands, A.N. Slavin, Nature 443 (7110), 430 (2006).

89. Y.D. Kalafati, V.L. Safonov, Sov. Phys. JETP 68 (6), 1162 (1989).

90. B. Hillebrands, Modern Techniques for Characterizing Magnetic Materials, Y. Zhu, Ed. (Kluwer Acad., Boston, 2005).

91. A.A. Serga, V.S. Tiberkevich, C.W. Sandweg, V.I. Vasyuchka, D.A. Bozhko, A.V. Chumak, T. Neumann, B. Obry, G.A. Melkov, A.N. Slavin, B. Hillebrands, Nat. Commun. 5, 3452 (2014).

92. S. Levy, E. Lahoud, I. Shomroni, J. Steinhauer, Nature 449 (7162), 579 (2007). 
93. K. Nakata, K.A. van Hoogdalem, P. Simon, D. Loss, Phys. Rev. B Condens. Matter 90 (14), 144419 (2014).

94. S.A. Bender, R.A. Duine, Y. Tserkovnyak, Phys. Rev. Lett. 108 (24), 246601 (2012).

95. S.A. Bender, R.A. Duine, A. Brataas, Y. Tserkovnyak, Phys. Rev. B Condens. Matter 90 (9), 094409 (2014).

96. P. Clausen, D.A. Bozhko, V.I. Vasyuchka, G.A. Melkov, B. Hillebrands, A.A. Serga, Condens. Matter (2015), available at http://arxiv.org/abs/1503.00482.

97. K. Vogt, H. Schultheiss, S. Jain, J.E. Pearson, A. Hoffmann, S.D. Bader, B. Hillebrands, Appl. Phys. Lett. 101 (4), 042410 (2012).

98. K. Vogt, F.Y. Fradin, J.E. Pearson, T. Sebastian, S.D. Bader, B. Hillebrands, A. Hoffmann, H. Schultheiss, Nat. Commun. 5, 3727 (2014).

99. J.Y. Gu, C.Y. You, J.S. Jiang, J. Pearson, Y.B. Bazaliy, S.D. Bader, Phys. Rev. Lett. 89 (26), 267001 (2002).

100. V. Pena, Z. Sefrioui, D. Arias, C. Leon, J. Santamaria, J.L. Martinez, S.G.E. te Velthuis, A. Hoffmann, Phys. Rev. Lett. 94 (5), 57002 (2005).

101. I.C. Moraru, W.P. Pratt, N.O. Birge, Phys. Rev. Lett. 96 (3), 37004 (2006).

102. J.E. Villegas, C.P. Li, I.K. Schuller, Phys. Rev. Lett. 99 (22), 227001 (2007).

103. G.-X. Miao, K.S. Yoon, T.S. Santos, J.S. Moodera, Phys. Rev. Lett. 98 (26), 267001 (2007).

104. C. Visani, P.J. Metaxas, A. Collaudin, B. Calvet, R. Bernard, J. Briatico, C. Deranlot, K. Bouzehouane, J.E. Villegas, Phys. Rev. B Condens. Matter 84 (5), 54539 (2011).

105. B. Li, N. Roschewsky, B.A. Assaf, M. Eich, M. Epstein-Martin, D. Heiman, M. Munzenberg, J.S. Moodera, Phys. Rev. Lett. 110 (9), 097001 (2013).

106. L.Y. Zhu, Y.H. Liu, F.S. Bergeret, J.E. Pearson, S.G.E. te Velthuis, S.D. Bader, J.S. Jiang, Phys. Rev. Lett. 110 (17), 177001 (2013).

107. N. Banerjee, C.B. Smiet, R.G.J. Smits, A. Ozaeta, F.S. Bergeret, M.G.

Blamire, J.W.A. Robinson, Nat. Commun. 5, 3048 (2014).

108. D. Stamopoulos, E. Manios, M. Pissas, Phys. Rev. B Condens. Matter 75 (1), 14501 (2007).

109. J. Zhu, X. Cheng, C. Boone, I.N. Krivorotov, Phys. Rev. Lett. 103 (2), 27004 (2009). 
110. A.A. Jara, C. Safranski, I.N. Krivorotov, C.T. Wu, A.N. Malmi-Kakkada, O.T. Valls, K. Halterman, Phys. Rev. B Condens. Matter 89 (18), 184502 (2014).

111. M.G. Flokstra, T.C. Cunningham, J. Kim, N. Satchell, G. Burnell, P.J. Curran, S.J. Bending, C.J. Kinane, J.F.K. Cooper, S. Langridge, A. Isidori, N. Pugach, M. Eschrig, S.L. Lee, Phys. Rev. B Condens. Matter 91 (6), 060501 (2015).

112. N. Poli, J.P. Morten, M. Urech, A. Brataas, D.B. Haviland, V. Korenivski, Phys. Rev. Lett. 100 (13), 136601 (2008).

113. C.H.L. Quay, D. Chevallier, C. Bena, M. Aprili, Nat. Phys. 9 (2), 84 (2013).

114. T. Wakamura, N. Hasegawa, K. Ohnishi, Y. Niimi, Y. Otani, Phys. Rev. Lett. 112 (3), 036602 (2014).

115. M. Eschrig, Phys. Today 64 (1), 43 (2011).

116. J. Linder, J.W.A. Robinson, Nat. Phys. 11 (4), 307 (2015).

117. N. Banerjee, J.W.A. Robinson, M.G. Blamire, Nat. Commun. 5, 4771 (2014). 
Table I. Selected effects where mesoscopic length scales play a determining role are summarized.

\begin{tabular}{llllll}
\hline \hline Effect & System & Measurements & Length scales & Phenomenon & Ref. \\
\hline Bohm-Aharonov & Rings & $\mathrm{MT}$ & $l(\mathbf{S}, \mathbf{M})$ & Topology & $3-5$ \\
Dimensional crossover & Superlattices & $\mathrm{H}_{\mathrm{C} 2}$ & $\xi(\mathbf{S})$ & Proximity & 6,7 \\
Solitons & Pillars & $\mathrm{FMR}$ & $d_{w}(\mathbf{M})$ & Topology & 8,9 \\
Collective pinning & Films/arrays & $\mathrm{MT}$ & $\lambda(\mathbf{S}, \mathbf{M})$ & Topology & 10 \\
Josephson & Trilayers & $\mathrm{I}-\mathrm{V}$ & $\xi(\mathbf{S})$ & Topology & 11 \\
Andreev reflection & Hetero & $\mathrm{I}-\mathrm{V}$ & $\xi, d_{s d}(\mathbf{S}, \mathbf{M})$ & Proximity & 12 \\
Vortex & Dots & $\mathrm{SPM}$ & $d_{e x}(\mathbf{M})$ & Topology & 13,14 \\
Skyrmion & Films & $\mathrm{SPM}$ & $d_{e x}(\mathbf{M})$ & Topology & 15,16 \\
Magnon bands & Superlattices & $\mathrm{BLS}$ & $d_{p}(\mathbf{M})$ & Dimensional & 17 \\
& & & & & \\
\hline
\end{tabular}

Note: MT, Magnetotransport; SPM, scanning probe microscopy; BLS, Brillouin light scattering; $H_{\mathrm{c} 2}$, upper critical magnetic field; FMR, ferromagnetic resonance; $l$, electronic mean free path; $\xi$, coherence length; $d_{\mathrm{w}}$, domain walls; $\lambda$, penetration depth; $d_{\mathrm{sd}}$, spin diffusion length; $d_{\mathrm{ex}}$, exchange length; $d_{\mathrm{p}}$, dipolar length(); I, current; V, voltage; $\mathrm{S}$, superconducting; $\mathrm{M}$, magnetic. 


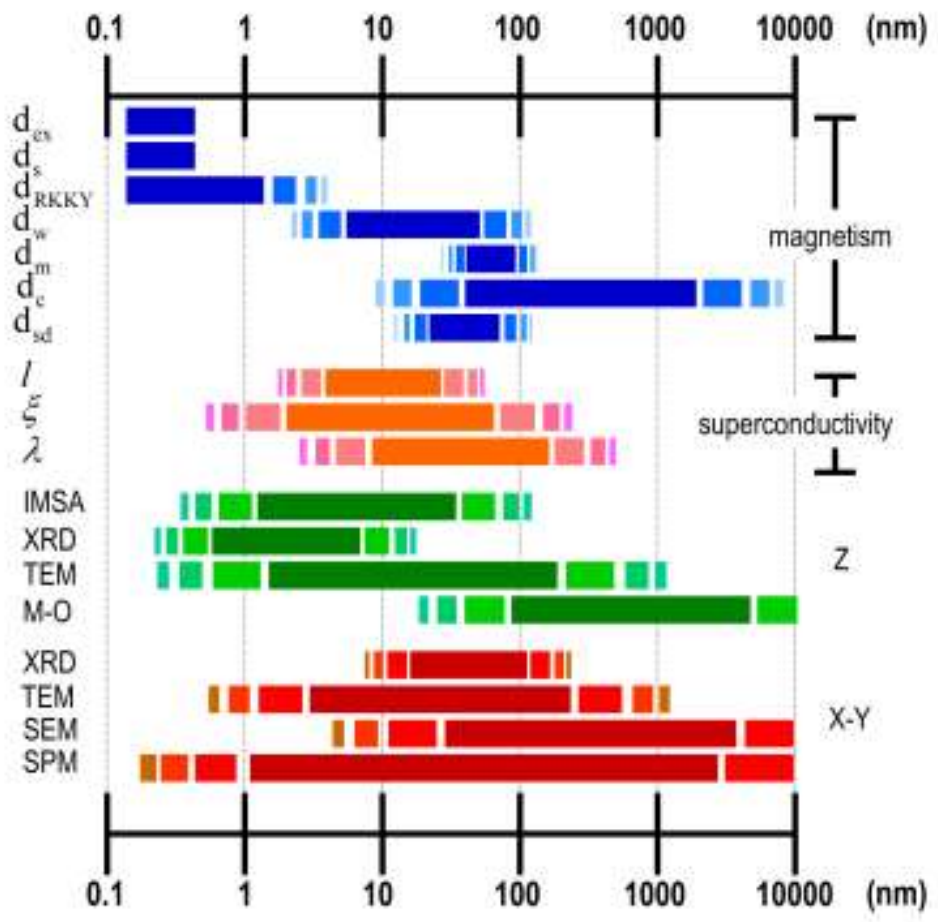

Figure 1. Comparison of selected characterization techniques used for mesoscopic magnetism and superconductivity with relevant length scales. $d_{\mathrm{ex}}=$ exchange length, $d_{\mathrm{s}}=$ screening length, $d_{\mathrm{R} \text { КК }}=$ Ruderman-Kittel-Kasuya-Yosida length, $d_{\mathrm{w}}=$ domain walls, $d_{\mathrm{m}}=$ magnetic dipolar length, $d_{\mathrm{c}}=$ magnetic domains, $d_{\mathrm{sd}}=$ spin diffusion length, $l=$ mean free path, $\xi=$ superconducting coherence length, $\lambda=$ superconducting penetration length, IMSA $=$ ion mill surface analysis, $\mathrm{XRD}=\mathrm{x}$-ray diffraction, $\mathrm{TEM}=$ transmission electron microscopy, $\mathrm{M}-\mathrm{O}=$ magneto-optics, SEM = scanning electron microscopy, SPM = scanning probe microscopy, $\mathrm{z}$ is the direction perpendicular to a layered system, and $\mathrm{x}-\mathrm{y}$ are the directions within the plane of a layered system. Adapted with permission from Reference 1, copyright (1999), Elsevier. 
(a)

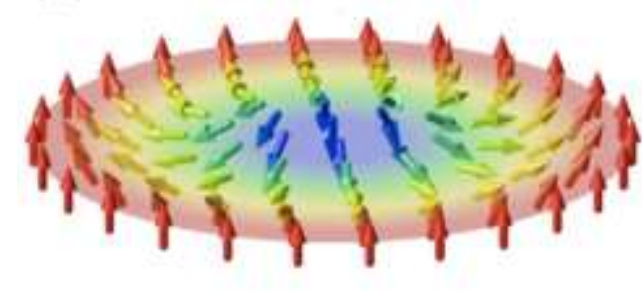

(b)

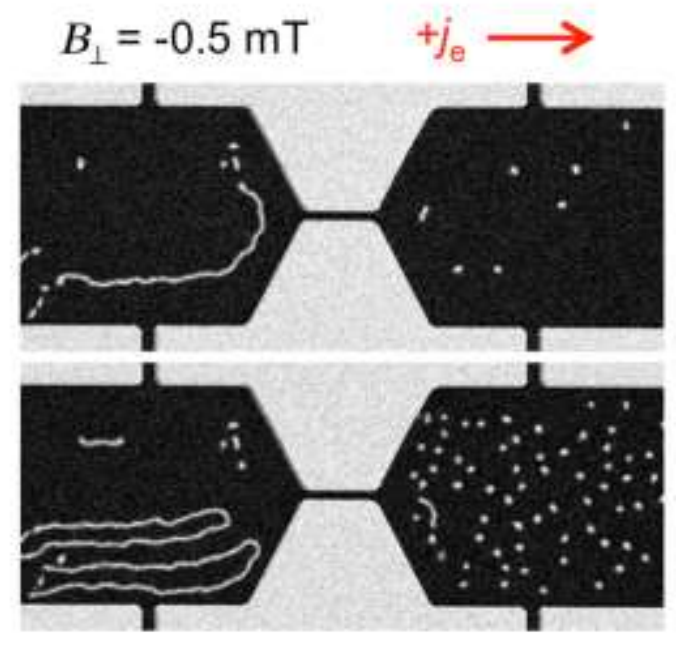

Figure 2. Magnetic skyrmions. (a) Schematic showing a skyrmion spin structure as expected from interfacial Dzyaloshinskii-Moriya interactions in magnetic multilayers. The color is used to emphasize the different out-of-plane magnetization components. (b) Magneto-optic Kerr effect (MOKE) imaging of the domain structure in a $\mathrm{Ta} / \mathrm{CoFeB} / \mathrm{TaO}_{x}$ trilayer patterned into a $60-\mu \mathrm{m}$ wide wire with a $3-\mu \mathrm{m}$ wide constriction. With a small magnetic field $\left(B_{\perp}\right)$ of $-0.5 \mathrm{mT}$ applied perpendicular to the film plane, only isolated band (left) and bubble domains (right) are visible. (c) MOKE imaging after a current pulse of $j_{e}=5 \times 10^{5}$ $\mathrm{A} / \mathrm{cm}^{2}$ has been applied. This results in the transformation of band domains on the left into magnetic skyrmions on the right. Adapted with permission from (a) Reference 23, copyright (2014), AIP Publishing LLC; and (b-c) Reference 15, copyright (2015), AAAS. 

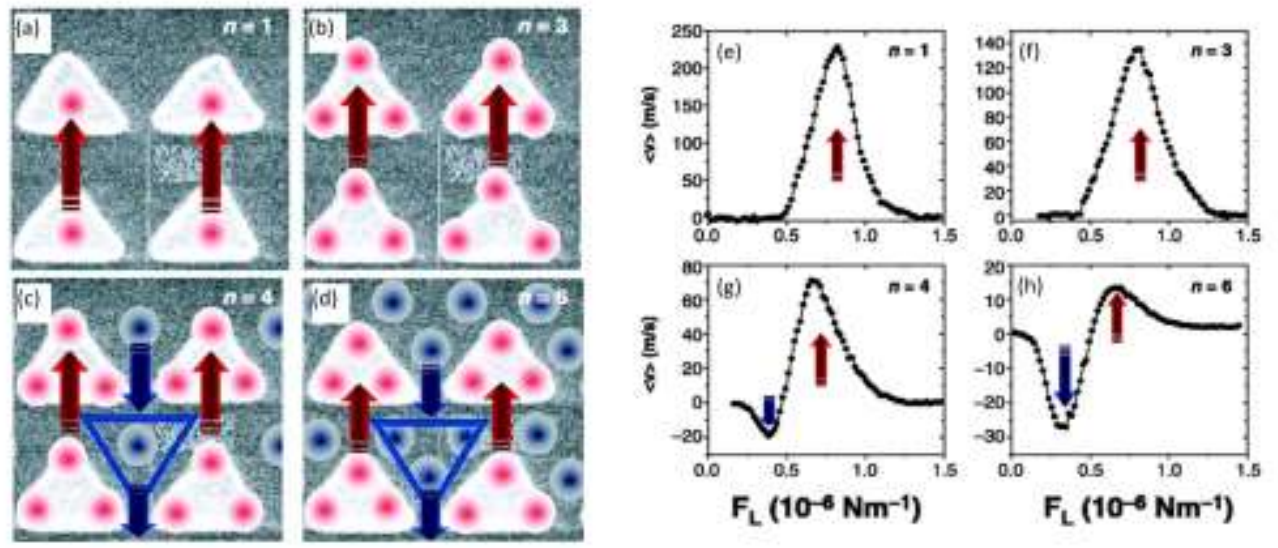

Figure 3. Superconducting vortex ratchets. (a-d) Sketches of the vortex configuration and net direction of motion (depicted with red and blue arrows) in $\mathrm{Nb}$ deposited over a magnetic nano-triangle pinning array, for different vortex densities. Vortices pinned on the triangles are shown in red and interstitial vortices in blue spheres. The distance between bottom sides of the triangles (white line) is $746 \mathrm{~nm}$. (e-h) Net vortex velocity $(\langle v\rangle)$ as a function of the AC drive amplitude $\left(F_{\mathrm{L}}\right)$ for the situations depicted in $(\mathrm{a}-\mathrm{d}) . n$ indicates the number of vortices per unit cell of the array. Adapted with permission from Reference 32, copyright (2003), AAAS. 

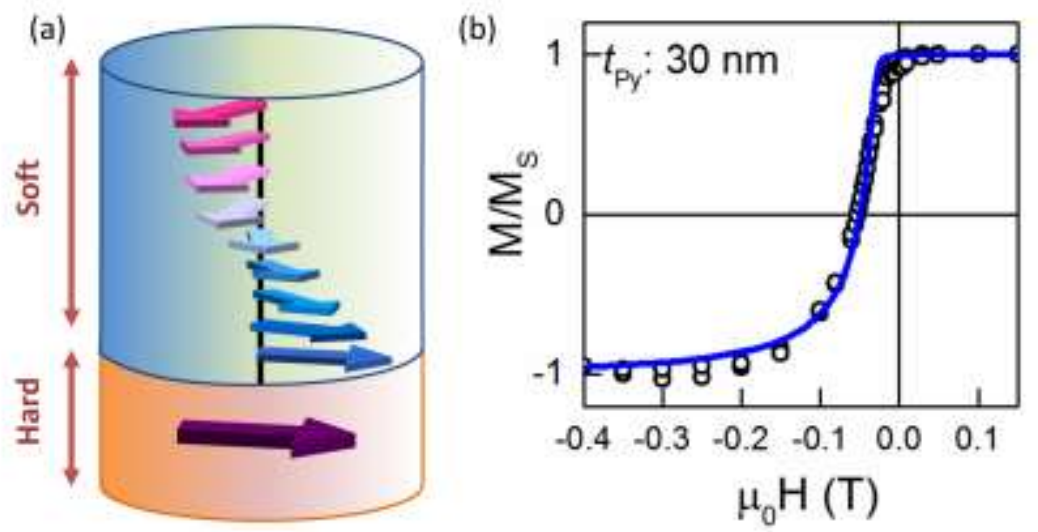

Figure 4. Exchange spring. (a) Depicted depth profile of the magnetization in the soft ferromagnetic layer during reversal. Colored arrows are to highlight different amount of rotations of moments from the pinning direction. (b) Example hysteresis curves of $\mathrm{Py} / \mathrm{FeF}_{2}$ bilayer exchange bias system that exhibits asymmetric and reversible behaviour due to exchange spring formation. Note: $M$, magnetization; $M_{\mathrm{s}}$, saturation magnetization; $H$, external magnetic field; $t_{\mathrm{Py}}$, thickness of permalloy layer. Adapted with permission from Reference 46, copyright (2015), American Physical Society. 
(a)

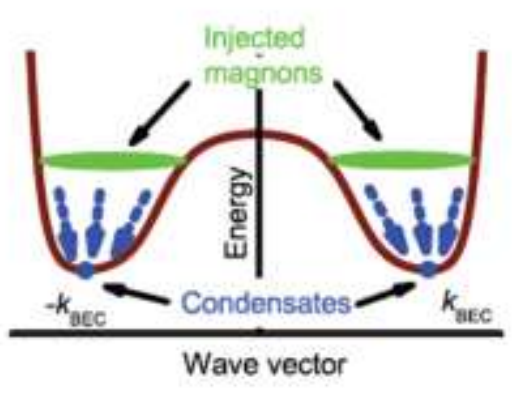

(b)

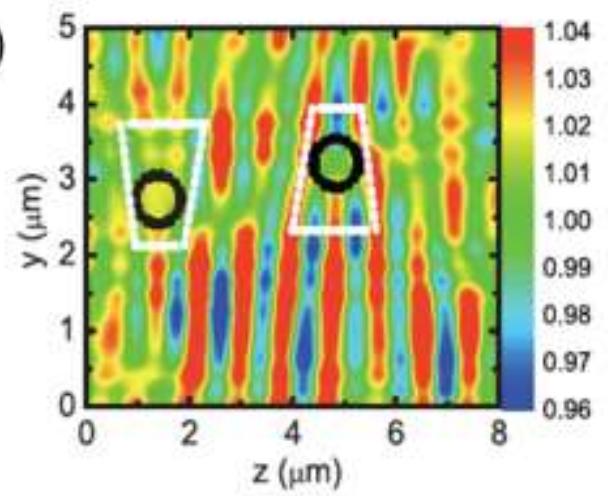

Figure 5: Bose-Einstein condensed magnons. (a) Schematic illustration of the magnon dispersion in a magnetic thin film. The competition between short-range exchange and long-range dipolar interactions gives rise to two global minima in the dispersion at opposite wave vectors. Magnons excited at higher energy can subsequently create two coherent Bose-Einstein condensates at the wavevectors $k_{\mathrm{BEC}}$ and $k_{\mathrm{BEC}}$, where the magnon dispersion has two minima. (b) Measurement of the spatial distribution of Bose Einstein condensed magnons in a $\mathrm{Y}_{3} \mathrm{Fe}_{5} \mathrm{O}_{12}$ thin film. The two magnon-condensates can interfere, which gives rise to a standing wave pattern. This can be directly probed with spatially resolved Brillouin lightscattering (BLS) measurements along the two lateral directions ( $\mathrm{y}$ and $\mathrm{z}$ ), where the scattered intensity (indicated by the color code on the right side of the graph) is proportional to the condensate density. Dashed areas and circles indicate the position of topological defects in the condensate. Adapted with permission from Reference 87, copyright (2012), Macmillan Publisher Ltd.. 

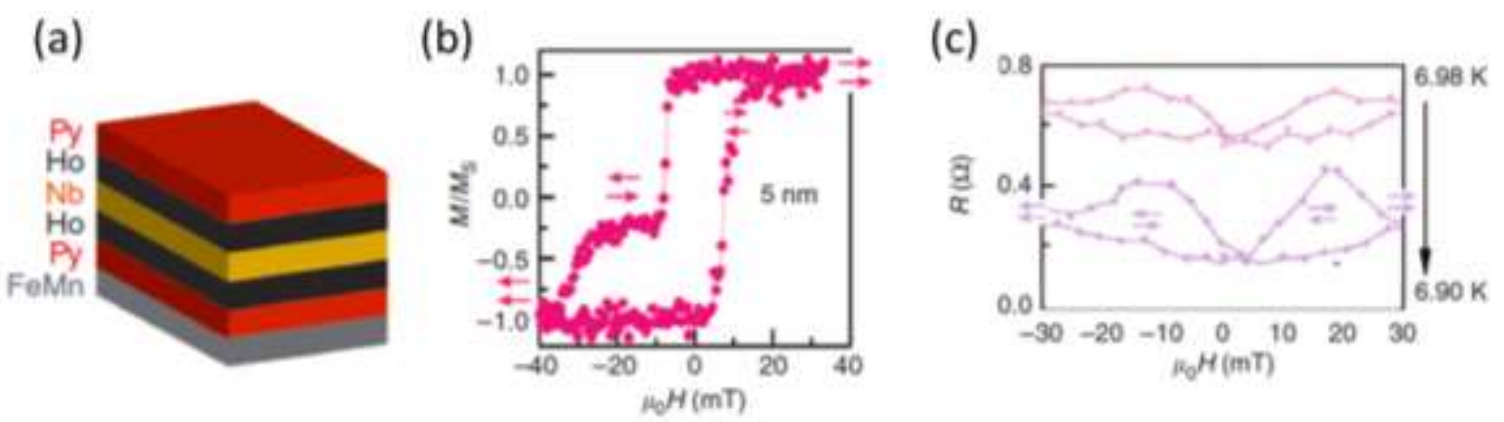

Figure 6. Superconducting spintronics. (a) Illustration of a triplet "superconducting spin valve". The superconductor $(\mathrm{Nb})$ is sandwiched between two ferromagnetic (FM) (Ho/Py bilayers). Ho is a conical magnet that behaves as a spin-mixer to generate equal-spin triplet state. (b) The hysteresis loop for a spin valve with a 5-nm-thick layer of Ho on either side of $\mathrm{Nb}$ shows that the exchange bias exerted by the FeMn layer on the lower Py layer, allows for parallel/antiparallel magnetization states of the Py layers. (c) The spin-valve magneto-resistance $(R)$ shows hysteresis, with a resistive switching within the field range in which the Py layers magnetization is labeled antiparallel. In the case of antiparallel magnetization, some of the triplet pairs are not be able to flow from one FM electrode into the opposite one, which results in higher resistance. The effect is observed only in a narrow (few $\mathrm{mK}$ ) temperature range across the superconducting transition $\left(\mathrm{T}_{\mathrm{c}} \sim 6.90 \mathrm{~K}\right)$. The curves correspond to two measurements at different temperatures within the range 6.98 to $6.90 \mathrm{~K}$. Note: $M$, magnetization; $M \mathrm{~s}$, saturation magnetization; $\mu$, magnetic permeability of free space; $H$, applied magnetic field. Adapted with permission from Reference 107, copyright (2014), MacMillan Publishers Ltd. 